\title{
Enhanced Influenza Surveillance using Telephone Triage Data in the VA ESSENCE Biosurveillance System
}

\author{
Cynthia A. Lucero-Obusan*1, Carla A. Winston ${ }^{1}$, Patricia L. Schirmer ${ }^{1}$, Gina Oda ${ }^{1}$, \\ Anoshiravan Mostaghimi ${ }^{1}$ and Mark Holodniy ${ }^{1,2}$
}

'Department of Veterans Affairs, Office of Public Health, Washington, DC, USA; ${ }^{2}$ Division of Infectious Diseases \& Geographic Medicine, Stanford University, Stanford, CA, USA

\section{Objective}

To evaluate the utility and timeliness of telephone triage (TT) for influenza surveillance in the Department of Veterans Affairs (VA).

\section{Introduction}

Telephone triage is a relatively new data source available to biosurveillance systems. ${ }^{1-2}$ Because early detection and warning is a high priority, many biosurveillance systems have begun to collect and analyze data from non-traditional sources [absenteeism records, overthe-counter drug sales, electronic laboratory reporting, internet searches (e.g. Google Flu Trends) and TT]. These sources may provide disease activity alerts earlier than conventional sources. Little is known about whether VA telephone program influenza data correlates with established influenza biosurveillance.

\section{Methods}

Veterans phoning VA's TT system, and those admitted or seen at a VA facility with influenza or influenza-like-illness (ILI) diagnosis were included in this analysis. Influenza-specific ICD-9-CM coded emergency department (ED) and urgent care (UC) visits, hospitalizations, TT calls, and ILI outpatient visits were analyzed covering 2010-2011 and 2011-2012 influenza seasons (July 11, 2010-April 14, 2012). Data came from 80 VA Medical Centers and over 500 outpatient clinics with complete reporting data for the time period of interest. We calculated Spearman rank-order coefficients, 95\% confidence intervals and p-values using Fisher's z transformation to describe correlation between TT data and other influenza healthcare measures. For comparison of time trends, we plotted data for hospitalizations, ED/UC visits and outpatient ILI syndrome visits against TT encounters. We applied ESSENCE detection algorithms to identify high-level alerts for influenza activity. ESSENCE aberration detection was restricted to the 2011-2012 season because limited historical TT and outpatient data from 2009-2010 was available to accurately predict aberrancy in the 2010-2011 season. We then calculated the peak measure of healthcare utilization during both influenza seasons (2010-2011 and 2011-2012) for each data source and compared timing of peaks and alerts between TT and other healthcare encounters to assess maximum healthcare system usage and timeliness of surveillance.

\section{Results}

There were 7,044 influenza-coded calls, 564 hospitalizations, 1,849 emergency/urgent visits, and 416,613 ILI-coded outpatient visits. Spearman rank correlation coefficients were calculated for influenza-coded calls with hospitalizations (0.77); ED/UC visits (0.85); and ILI-outpatient visits $(0.88)$, respectively $(\mathrm{P}<0.0001$ for all correlations). Peak influenza activity occurred on the same week or within 1 week across all settings for both seasons. For the 2011-2012 season, TT alerted with increased influenza activity before all other settings.

\section{Conclusions}

Data from VA telephone care correlates well with other VA data sources for influenza activity. TT may serve to augment these existing clinical data sources and provide earlier alerts of influenza activity. As a national health care system with a large patient population, VA could provide a robust early-warning system for influenza if ongoing biosurveillance activities are combined with TT data. Additional analyses are needed to understand and correlate TT with healthcare utilization and severity of illness.

\section{Keywords}

Surveillance; Influenza; Telephone triage; Veterans

\section{References}

1. Yih WK, Teates KS, Abrams A, Kleinman K, Kulldorff M, Pinner R, Harmon R, Wang S, Platt R: Telephone triage service data for detection of influenza-like illness. PLoS One 2009, 4(4):e5260.

2. van Dijk A, McGuinness D, Rolland E, Moore KM: Can Telehealth Ontario respiratory call volume be used as a proxy for emergency department respiratory visit surveillance by public health? CJEM 2008, $10(1): 18-24$

*Cynthia A. Lucero-Obusan

E-mail: cynthia.lucero@va.gov 